This item was submitted to Loughborough's Research Repository by the author.

Items in Figshare are protected by copyright, with all rights reserved, unless otherwise indicated.

\title{
High-intensity demands of 6-a-side small-sided games and 11-a-side matches in youth soccer players
}

PLEASE CITE THE PUBLISHED VERSION

https://doi.org/10.1123/pes.2018-0122

\section{PUBLISHER}

(c) Human Kinetics

\section{VERSION}

AM (Accepted Manuscript)

\section{PUBLISHER STATEMENT}

This work is made available according to the conditions of the Creative Commons Attribution-NonCommercialNoDerivatives 4.0 International (CC BY-NC-ND 4.0) licence. Full details of this licence are available at: https://creativecommons.org/licenses/by-nc-nd/4.0/

\section{LICENCE}

CC BY-NC-ND 4.0

\section{REPOSITORY RECORD}

Goto, Heita, and James King. 2019. "High-intensity Demands of 6-a-side Small-sided Games and 11-a-side Matches in Youth Soccer Players". figshare. https://hdl.handle.net/2134/35427. 
Title:

High-intensity demands of 6-a-side small-sided games and 11-a-side matches in youth soccer players

Running title:

Small-sided games and matches in youth soccer

Corresponding author:

Heita Goto

Faculty of Sports Science, Kyushu Kyoritsu University, , Japan

81-(0)93-693-3425

heitagoto@hotmail.com

Co-authors:

JA King

School of Sport, Exercise and Health Sciences, Loughborough University, UK

J.A.King@lboro.ac.uk 


\section{ABSTRACT}

2 Purpose. The purposes of the present study were to examine: high-intensity running distance

3 during 6-a-side small-sided games (SSGs) and 11-a-side matches (11M) in youth soccer

4 players using speed and metabolic power approaches and; the magnitude of difference

5 between high-intensity running distance calculated with the two approaches. Method. Eleven

6 outfield players (age $=16.3 \pm 0.6$ years) performed SSGs with three pitch sizes (small SSG

7 (SSGS), medium SSG (SSGM) and large SSG (SSGL)) and 11M. A Global Positioning

8 System $(15 \mathrm{~Hz})$ was employed to calculate total distance covered, distance covered at a speed

$9 \geq 4.3 \mathrm{~m} \cdot \mathrm{s}^{-1}(\mathrm{TS})$ and metabolic power of $\geq 20 \mathrm{~W} \cdot \mathrm{kg}^{-1}$ (TP). Results. The total distance

10 covered increased from SSGS through to SSGL $(\mathrm{P}<0.001)$ and was greater during $11 \mathrm{M}$ and

11 SSGL compared to other SSGs $(\mathrm{P}<0.01)$. TS and TP increased from SSGS (TS vs. TP = $98 \pm$ 1255 vs. $547 \pm 181 \mathrm{~m})$ through to SSGL (538 \pm 167 vs. $1050 \pm 234 \mathrm{~m}, \mathrm{P}<0.001)$. TS and TP 13 during $11 \mathrm{M}(370 \pm 122$ vs. $869 \pm 233 \mathrm{~m})$ was greater than SSGS $(\mathrm{P}<0.001$ for both $)$ and less 14 than SSGL ( $\mathrm{P}<0.05$ for both). The magnitude of difference between TS and TP (\%) reduced 15 with an increase in pitch size during SSGs and was greater in SSGS $(615 \pm 404 \%, \mathrm{P}<0.001)$ and SSGM (195 \pm 76\%, $\mathrm{P}<0.05)$ and smaller in SSGL $(102 \pm 33 \%, \mathrm{P}<0.01)$ compared to $11 \mathrm{M}(145 \pm 53 \%)$. Conclusion. SSGs can replicate the high-intensity demands of $11 \mathrm{M}$ and the speed approach underestimates high-intensity demands of SSGs and 11M compared to the metabolic power approach. 


\section{INTRODUCTION}

Small-sided games (SSGs) have been commonly used as a training drill by coaches to develop physical fitness $(21,25,35)$ or technical and tactical abilities $(27,32,36)$ of soccer players.

Many studies have investigated the variables which influence the physical demands during SSGs (23) and such variables include pitch size (area per player) $(7,34)$, player number $(1,3,22$,$) , coach feedback (2,34)$, training regimen (continuous or interval) $(9,24)$, rule modifications (20), use of goals and/or goalkeepers (10) and prior knowledge of exercise duration (12). Hence, many elements must be considered to control the physical demands during SSGs.

The identification of training modalities which most closely replicate the physical demands of soccer match play is of great interest to coaches and exercise scientists who are concerned with optimizing training stimuli $(8,34)$. To date, only three studies have compared the demands of SSGs and 11-a-side matches $(11 \mathrm{M})(8,13,28)$. Unfortunately, within each of these previous studies more than one game-related variable has been manipulated (e.g. player number and pitch size) making it impossible to isolate the independent effect of either.

Furthermore, each of these previous studies examined adult participants, and it is recognized that players in the developing stages should not be considered as miniature adults (37). It is therefore necessary to examine the differences in the physical demands between SSGs and $11 \mathrm{M}$ in young soccer players whilst modifying only one variable to investigate the specific format of SSGs which mimic the physical demands of 11M.

In recent years, the metabolic power approach has been employed to examine the physical demands of training sessions $(15)$, SSGs $(14,39)$ and match play $(33)$ in elite professional soccer players; with a common use of Global Positioning Systems (GPS) for data collection 
$(14,15)$. Metabolic power is obtained by multiplying the estimated energy cost of accelerated/decelerated running on a horizontal level with an assumption that accelerated/decelerated running on a horizontal level is energetically equivalent to uphill/downhill running at a constant speed on an 'equivalent' slope. As energy costs are independent of the velocity and the energetics of uphill/downhill running can be described, an estimation of the energy costs of accelerated/decelerated running on a horizontal level can be achieved (33). The metabolic power approach involves accelerations and decelerations whereas the traditional speed approach only includes distances covered at constant speeds. The latter neglects the importance of accelerations and decelerations when estimating metabolic demands (33). Accelerations are a pre-cursor to running at high speeds and during accelerations, a greater neural activation to the working muscles and a higher rate of force production are required compared to a constant speed running $(29,33)$. Even when moving at low speeds, a great amount of metabolic load is imposed on soccer players when acceleration is raised, and decelerations occur as frequently as accelerations in soccer that each significantly contribute to the physical demands of soccer (33). Moreover, the metabolic power approach is more strongly related to energy expenditure compared to the traditional speed approach $(5,19)$.

The high-intensity demands during SSGs (14) and match-play (33) have previously been examined with speed and metabolic power approaches in professional soccer players. The previous study employed 5-a-side, 7-a-side and 10-a-side SSGs with area per player of 75, 98 and $135 \mathrm{~m}^{2}$, respectively (14). The results showed increases in high-intensity running distance with increases in player number and area per player in both approaches. Moreover, the highintensity running distance of SSGs were underestimated by the speed approach compared to the metabolic power approach by around 45-350\% and the underestimation was greater when 
the area per player was reduced (14). In addition, the speed approach underestimated the highintensity running distance during a match by $\sim 45 \%$ in professional soccer players compared to the metabolic power approach (33). Although the high-intensity demands of SSGs and 11M have been investigated in the separate studies using two approaches (speed vs metabolic power), from the authors’ knowledge, such variables have not been examined in a single study with the same participants $(14,33,39)$. Furthermore, metabolic power related data on SSGs and $11 \mathrm{M}$ is only available on senior and elite youth players $(14,33,39)$. Since physiological and physical responses during SSGs and 11M differs depending on standard of play (30,39), an investigation of the high-intensity demands of SSGs and 11M using two approaches (speed vs metabolic power) in non-elite youth players would provide a greater understanding of relationships between the two approaches (speed vs metabolic power) during SSGs and 11M in this particular category of players. Such investigation would support coaches and sports scientists to provide group specific training programs and maximize performance enhancement.

Therefore, the aims of the present study were to examine: 1) the high-intensity demands of SSGs and 11M in youth soccer players using two approaches (speed vs metabolic power) and: 2) the magnitude of difference between running distance calculated with speed and metabolic power approaches during SSGs and 11M. 
95

96

97

98

99

100

101

102

103

104

105

106

107

108

109

110

111

112

113

114

115

116

117

118

\section{METHOD}

\section{Participants}

The subjects were 11 outfield players from the same soccer team who competed in regional level competitions (age $=16.3 \pm 0.6$ years; height $=170.1 \pm 6.4 \mathrm{~cm}$; body mass $=59.8 \pm 7.5$ kg; playing experience $=6.1 \pm 1.3$ years; $10 \mathrm{~m}$ sprint time $=1.74 \pm 0.08 \mathrm{~s}$; Yo-Yo intermittent recovery test level $1=1316 \pm 289 \mathrm{~m}$ ). The team trained five times and played one match per week on average during a season and all training involved technical based sessions. Subjects were provided with a written and verbal explanation of the study including experimental protocols and all measurements to be taken. Each player signed an informed assent form and completed a health screen questionnaire prior to participation in the study. Each player's parent signed a consent form prior to the start of the study. Players were free to withdraw from the study without giving any reasons. The study was approved by a University Ethical Committee.

\section{6-a-side small-sided games and 11-a-side matches}

The participants performed 6-a-side SSGs (five field players and a goalkeeper) with three different pitch sizes (small SSG (SSGS), medium SSG (SSGM), large SSG (SSGL)) and 11M as part of training sessions. Established characteristics of SSGs and 11M are shown in table 1 and all SSGs and 11M employed the same pitch length to width ratio. In SSGs, each team contained two central defenders, a defensive midfielder, a central attacking midfielder and a striker. A playing system of 4-2-3-1 was only allowed during 11M. Participants played their natural playing positions during SSGs and 11M. The players included in each team were generally fixed for all SSGs and 11M but there was a maximum of one player difference in a team in some sessions due to injuries or unavailability. The players in each team were 
119 selected by the coach who was asked to include players with similar ability to balance the 120 strength of the teams.

121

122 All data collection took place on the same pitch which was a third generation synthetic

123 astroturf (Grand Grass F-M DS, Mizuno corporation, Osaka, Japan). Laws of the game (40)

124 were applied during SSGs and 11M but the offside rule was neglected during SSGs. Each of

125 the SSGs and 11M were conducted four times during six weeks and two to three sessions took

126 place in each week. They were conducted in a counterbalanced order and the day after a

127 match was avoided. All participants took part in each of the SSGs and $11 \mathrm{M}$ for $2.7 \pm 0.8$ times

128 (range $=2-4$ times). Each session started with the same warm-up from 15:00 (approximately

12930 minutes) which involved static and dynamic stretches, running at various speeds from

130 jogging to sprinting and technical drills. The duration of all SSGs and 11M was 35 minutes

131 because that was the duration of a half of participants' official matches. A multi-ball system

132 was employed to minimise non-playing time and similar verbal encouragement was given by

133 the coach during SSGs and 11M as coaches' feedback can influence physical demands $(2,34)$.

134 The environmental temperature was between 24 and $28{ }^{\circ} \mathrm{C}$ and humidity between 63 and $85 \%$

135 during the data collections (rainy days were avoided).

136

137 Table 1 here

\section{Physical demands}

140 The previously reported equation has been employed to estimate metabolic power and

141 assumed energy cost of running at constant speed was $3.6 \mathrm{~J} \cdot \mathrm{kg}^{-1} \cdot \mathrm{m}^{-1}$ (33).

$143 \quad$ Metabolic power $=\mathrm{EC} \cdot \mathrm{v}$ 
145 Where, $\mathrm{EC}=$ the energy cost of accelerated running on grass $\left(\mathrm{J} \cdot \mathrm{kg}^{-1} \cdot \mathrm{m}^{-1}\right)=\left(155.4 \cdot \mathrm{ES}^{5}-\right.$

$\left.146 \quad 30.4 \cdot \mathrm{ES}^{4}-43.3 \cdot \mathrm{ES}^{3}+46.3 \cdot \mathrm{ES}^{2}+19.5 \cdot \mathrm{ES}+3.6\right) \cdot \mathrm{EM} \cdot \mathrm{KT}, \mathrm{ES}=$ the equivalent slope $=\tan (90$

$\left.147-\arctan \mathrm{g} / \mathrm{a}_{\mathrm{f}}\right), \mathrm{g}=$ Earth's acceleration of gravity; $\mathrm{a}_{\mathrm{f}}=$ forward acceleration; $\mathrm{EM}=$ the

148 equivalent body mass $=\left[\left(\mathrm{af}_{\mathrm{f}}^{2} \cdot \mathrm{g}^{-2}\right)+1\right]^{0.5}, \mathrm{KT}=\mathrm{a}$ constant $=1.29, \mathrm{v}=\operatorname{running}$ speed $\left(\mathrm{m} \cdot \mathrm{s}^{-1}\right)$.

In addition to total distance covered, high-intensity physical demands were analysed with the assessment of speed and metabolic power. The distance covered at a speed $\geq 4.3 \mathrm{~m} \cdot \mathrm{s}^{-1}$ (TS) (33) and metabolic power of $\geq 20 \mathrm{~W} \cdot \mathrm{kg}^{-1}$ (TP) $(14,15)$ were calculated. These values were chosen because $20 \mathrm{~W} \cdot \mathrm{kg}^{-1}$ is the metabolic power when running at a constant speed of approximately $4.3 \mathrm{~m} \cdot \mathrm{s}^{-1}$ on natural (33) and artificial (38) grass. Physical demands were analysed with $15 \mathrm{~Hz}$ (5 Hz signal interpolated to $15 \mathrm{~Hz}$ ) GPS technology (SPI HPU, GPSports, Canberra, Australia) which was positioned on the upper back in a custom-made vest. This particular device has been reported to possess less than $1 \%$ error in estimating the total distance covered when 8 laps of a team sport simulation circuit (165 m) was completed. The circuit included resting, different type of movements (straight walking/running, figure eight agility run, 90 degrees turning), various speeds (walking to sprinting) and fast accelerations/decelerations (26). Moreover, there was a $<5 \%$ difference in maximal speed during 30 m sprint with split times at 10 and 20 m between the values estimated using GPS and photoelectric timing gates (26). Moreover, inter-unit reliability (percentage typical error

164 of measurement) for total distance covered, distance covered at $<3.9 \mathrm{~m} \cdot \mathrm{s}^{-1}, 3.9-5.6 \mathrm{~m} \cdot \mathrm{s}^{-1}$ and $165>5.6 \mathrm{~m} \cdot \mathrm{s}^{-1}$ were $1.9,2.0,7.6$ and $12.1 \%$, respectively (26). At least 8 satellites (mean $\pm \mathrm{SD}=$ $9.5 \pm 0.8$ satellites) were connected during data collection which is the minimum number of satellites required to allow an accurate measurement $(41,42)$ and mean horizontal dilution of position was $1.2 \pm 0.2$ during data collections. Total distance covered, TS and TP were 
169 calculated using Team AMS software version R1.2016.4 (GPSports, Canberra, Australia) and

170 the software filtered through all data concerning velocity, acceleration and deceleration to

171 eliminate noise before calculating the distance.

\section{Statistical analyses}

173 The mean values from SSGs and 11M for each player were calculated before calculating

174 group means and conducting the statistical analyses. The magnitude of difference was

175 calculated by dividing an absolute difference between TS and TP by TS and multiplied by

176 100. Data were normally distributed as examined by a Kolmogorov-Smirnov test. Levene’s

177 Test revealed that variances were unequal for SSGs and 11M. Hence, one-way analysis of

178 variance with Games-Howell post hoc test was employed to compare physical demands

179 between $11 \mathrm{M}$ and SSGs. The effect size $\left(\eta^{2}\right)$ was calculated and values of $0.01,0.06$ and

180 above 0.15 were considered as small, medium and large, respectively (11). Levene’s Test

181 revealed that variances were equal for TS and TP in each of the SSGs and 11M therefore an

182 independent sample t-test was employed to assess whether or not there were statistically

183 significant differences between TS and TP. The effect sizes $(d)$ for these differences were

184 calculated as (mean A - mean B)/ (pooled SD) and values of 0.2, 0.5 and above 0.8 were

185 considered to represent small, moderate and large differences, respectively (11). The level of

186 statistical significance was set at $\mathrm{p}<0.05$. Results are presented as mean \pm standard deviation

187 (SD) and IBM SPSS 22.0 was used for all the statistical analyses. 


\section{RESULTS}

189

190

191

192

193

194

195

196

197

198

199

200

201

202

203

204

205

206

207

208

209

210

\section{Comparison of physical demands between SSGs}

Total distance covered, TS and TP increased from SSGS through to SSGL (P $<0.001$ for all, $\left.\eta^{2}=0.44-0.65\right)$ (figure 1-3).

Figure 1 to 3 here

\section{Comparison of physical demands between SSGs and 11M}

The total distance covered during $11 \mathrm{M}$ was similar to SSGL and greater than SSGS and SSGM (P $<0.001$ for all, $\eta^{2}=0.58$ ) (figure 1$)$. TS during $11 \mathrm{M}$ was approximately four times greater than SSGS $(\mathrm{P}<0.001)$ and $\sim 45 \%$ less than SSGL $(\mathrm{P}<0.001)\left(\eta^{2}=0.65\right)$ (figure 2$)$. TP during $11 \mathrm{M}$ was $\sim 59 \%$ greater than SSGS $(\mathrm{P}<0.001)$ and $\sim 21 \%$ less than SSGL $(\mathrm{P}<$ $0.05)\left(\eta^{2}=0.44\right)$ (figure 3$)$.

\section{Difference between TS and TP}

TP was greater than TS in all SSGs and 11M ( 450 to $\sim 520 \mathrm{~m}, \mathrm{P}<0.001, d=1.3-1.9$ for all) (figure 2 and 3). The magnitude of difference between TS and TP (\%) reduced with an increase in pitch size from $\sim 620 \%$ for SSGS to $~ 100 \%$ for SSGL $\left(\mathrm{P}<0.001, \eta^{2}=0.51\right)$ (figure 4). Moreover, the magnitude of difference between TS and TP (\%) in 11M was greater than SSGL $(\mathrm{P}<0.01)$ and less than SSGS $(\mathrm{P}<0.001)$ and SSGM $(\mathrm{P}<0.05)\left(\eta^{2}=0.51\right)$ (figure 4). 


\section{DISCUSSION}

212 This is the first study that examined the high-intensity demands of 6-a-side SSGs (three

213 different pitch sizes) and 11M using speed and metabolic power approaches in youth soccer

214 players. The main findings of the present study were that: 1) the high-intensity demands of 6-

215 a-side SSGs increased when the pitch size was enlarged regardless of approaches (speed vs

216 metabolic power); 2) TS and TP during 11M and SSGM were similar; 3) the speed approach

217 underestimated the high-intensity demands of 6-a-side SSGs and 11M compared to the

218 metabolic power approach; 4) the underestimation of high-intensity demands during SSGs

219 increased with a reduction in pitch size; and 5) the underestimation of high-intensity demands 220 during $11 \mathrm{M}$ was less than SSGM.

The first major finding of the current study was that total distance covered, TS and TP during 6-a-side SSGs, increased when pitch size was expanded. For total distance covered, a 224 previous study of 15-year-old boys during 6-a-side SSGs agreed with the current findings (7).

225 However, the previous study reported that TS was only greater in medium and large compared to small pitch size with no differences between medium and large pitches (7). This disagreement is possibly because the previous study employed a smaller pitch size ratio between medium and large SSGs compared to the current study (medium: large = current, 1: 2 vs previous, 1: 1.5) (7). Moreover, similar findings to the current study in TP have been demonstrated in professional soccer players when player number and area per player were increased together (14).

The total distance covered during SSGL and 11M in the current study was similar to the 234 previous studies. The current participants covered $\sim 4000 \mathrm{~m}$ during $11 \mathrm{M}$ and when the 235 distance was adjusted to match playing time, the distance was consistent with under-16 soccer 
236 players from England $(16,18)$ and Qatar (6). Moreover, the distance covered during $11 \mathrm{M}$ was

237 similar to SSGL which suggests that total distance does not differ when player number

238 changes as long as the area per player is the same. However, the previous studies reported that 239 a change in player number influences (22) or does not influence (1) total distance and total 240 distance is a poor indicator of global work rate in SSGs (22) and 11M (30).

242 The second major finding of the current study was that TS and TP during $11 \mathrm{M}$ were greater 243 than SSGS, less than SSGL and similar to SSGM. A previous study which examined the 244 physical demands of 6-a-side SSGs and 11M in semi-professional soccer players concluded 245 that SSGs are played at a higher intensity than 11M when area per player of SSGs was two246 thirds of $11 \mathrm{M}$ (SSG vs $11 \mathrm{M}=200$ vs $300 \mathrm{~m}^{2}$ ) (8). Conversely, the area per player of SSGM 247 was roughly half of $11 \mathrm{M}$ in the current study (SSGM vs $11 \mathrm{M}=165$ vs $325 \mathrm{~m}^{2}$ ). These 248 findings suggest that 6-a-side SSGs with roughly half the area per player of $11 \mathrm{M}$ provides a 249 similar high-intensity demand to 11M; whereas 6-a-side SSGs with around two-thirds and 250 greater area per player of $11 \mathrm{M}$ offer a greater high-intensity demand than $11 \mathrm{M}$. In addition, 251 players perform less high-intensity running during 6-a-side SSGs than 11M when area per 252 player of SSGs is approximately a quarter of $11 \mathrm{M}$.

254 The third major finding of the current study was that TP was greater than TS during all SSGs 255 and 11M. Similar findings have been reported during various SSGs (14,39), 11M (33) and 256 training sessions (15). In the current study, the magnitude of difference between TS and TP 257 during SSGs were $\sim 100$ to $\sim 620 \%$ and that was reduced with pitch size from SSGS through 258 SSGL. Similar values ( 20\% to 349\%) (14,39) and a trend (14) have been observed in the 259 previous studies on SSGs. However, the previous study modified player number and area per 260 player together (14) and the current study is the first to demonstrate that a modification of 
261

262

263

264

265

266

267

268

269

270

271

272

273

274

275

276

277

278

279

280

281

282

283

284

285

area per player alone still alters the same relationship between area per player and magnitude of difference between TS and TP in SSGs. The variations in the underestimation of highintensity demands in SSGs exists because players are required to produce a greater or lesser proportion of high-intensity activities at constant high speeds depending on pitch size of SSGs and that results in decreases or increases in the production of explosive accelerations and decelerations $(14,15)$. Although the metabolic power approach reflects metabolic internal loads, and running distance calculated by the speed approach demonstrates external loads, the employment of the metabolic power approach probably offers a more valid indication of the high-intensity demands of SSGs and 11M in youth soccer player as metabolic power approach includes demands of accelerations/decelerations (14).

A further major outcome of the current study was that the magnitude of difference between TS and TP during $11 \mathrm{M}$ was $~ 145 \%$. This value seems to be greater than the magnitude of difference reported from match play of professional soccer players ( 45\%) (33). The professional players covered a greater proportion of total match distance by high-intensity running (current vs professional: $4.3 \mathrm{~m} \cdot \mathrm{s}^{-1} \mathrm{vs} 4.4 \mathrm{~m} \cdot \mathrm{s}^{-1}$ ) compared to the participants in the current study (current vs professional: 9\% vs 18\%) (33) which suggests that the professional players produced a larger proportion of high-intensity activities at constant high speeds and a less amount of explosive accelerations and decelerations at low speeds compared to the participants of current study $(14,15)$. Hence, the speed approach underestimates high-intensity demands of match play compared to metabolic power approach especially in the players who covers less distance with high speeds.

Given that TS and TP did not differ between SSGM and 11M in the current study, the magnitude of difference between TS and TP was greater during SSGM compared 11M. The 
rationale for this finding is unknown but modification of player number alone has been shown to influence demands of SSGs including heart rate $(1,3,22)$ and running distance $(1,22)$ related variables that different number of players employed in SSGM and 11M may explain the current result. The current study mainly focused on the influence of pitch size modification on physical demands during SSGs with a fixed player number and future studies should investigate the influence of player number on high-intensity demands and the underestimation of speed approach compared to metabolic power approach.

There are three possible limitations to the current study. Firstly, the current study compared the high-intensity demands of SSGs and $11 \mathrm{M}$ with a playing time of 35 minutes in all sessions. However, a single bout of SSGs is traditionally much shorter than the duration employed in the current study (3-8 minutes) (17) and longer duration would reduce distance covered in high speeds and frequency of high intensity activities (24). Hence, it is important to note that an employment of SSGs with a different duration to the current study may result in players showing different physical responses. Secondly, the current study could not include heart rate (HR) in the analysis due to having invalid HR data in many occasions. HR data may have supported the analysis by providing physiological loads which is different to displacement measures. Finally, the current study did not control tactical aspects of SSGs and 11M. Although tactical differences can influence physical responses during SSGs (31) and $11 \mathrm{M}$ (4), this is an under researched area that such aspects may need to be explored further in the future research. 


\section{CONCLUSION}

310 The current findings demonstrate that 6-a-side SSGs can replicate the high-intensity demands

311 of $11 \mathrm{M}$ in youth soccer players when the area per player of the SSGs is approximately half of

$31211 \mathrm{M}$. On the other hand, two-thirds or greater area per player of $11 \mathrm{M}$ provides a greater high-

313 intensity demand during 6-a-side SSGs and a quarter of the area per player of 11M would

314 require less high-intensity demand during 6-a-side SSGs compared to 11M. Moreover, total

315 distance covered and high-intensity running distance during 6-a-side SSGs increase with

316 enlargement of pitch size regardless of approach (speed vs metabolic power). However, the

317 speed approach underestimates the high-intensity demands of SSGs and 11M compared to the

318 metabolic power approach; and the underestimation increases exponentially with a reduction

319 in pitch size in SSGs. Therefore, coaches and sports scientists should pay attention to

320 methodology for monitoring players (speed vs metabolic power) and are advised to carefully

321 choose pitch size of SSGs together with number of players per team depending on the aim of

322 training sessions. 


\section{REFERENCES}

325 1. Aguiar MV, Botelho GM, Goncalves BS, Sampaio, JE. Physiological responses and activity profiles of football small-sided games. J Strength Cond Res. 2013;27: 12871294.

2. Brandes M, Elvers, S. Elite youth soccer players’ physiological responses, time-motion characteristics, and game performance in 4 vs. 4 small-sided games: the influence of coach feedback. J Strength Cond Res. 2017;31:2652-2658.

3. Brandes M, Heitmann A, Muller L. Physical responses of different small-sided game formats in elite youth soccer players. J Strength Cond Res. 2012;26:1353-1360.

4. Bradley PS, Carling C, Archer D et al. The effect of playing formation on high-intensity running and technical profiles in English FA Premier League soccer matches. J Sports Sci. 2011;9: 821-830.

5. Buchheit, M, Manouvrier, C, Cassirame, J, Morin, JB. Monitoring Locomotor Load in Soccer: Is Metabolic Power, Powerful? Int J Sports Med. 2015;36:1149-1155.

6. Buchheit M, Mendez-Villanueva A, Simpson BM, Bourdon PC. Match running performance and fitness in youth soccer. Int J Sports Med. 2010;31:818-825.

7. Casamichana D, Castellano J. Time-motion, heart rate, perceptual and motor behaviour demands in small-sides soccer games: effects of pitch size. J Sports Sci. 2010;28:16151623.

8. Casamichana D, Castellano J, Castagna C. Comparing the physical demands of friendly matches and small-sided games in semi professional soccer players. J Strength Cond Res. 2012;26:837-843.

9. Casamichana D, Castellano J, Dellal A. Influence of different training regimes on physical and physiological demands during small-sided soccer games: continuous vs. intermittent format. J Strength Cond Res. 2013;27:690-697. 
10. Castellano J, Casamichana D, Dellal A. Influence of game format and number of players on heart rate responses and physical demands in small-sided soccer games. J Strength Cond Res. 2013;27:1295-1303.

11. Cohen J. Statistical Power Analysis for the Behavioral Sciences. Hillsdale, NJ: Lawrence Erlbaum Associates; 1988.

12. Ferraz R, Gonçalves B, Van Den Tillaar R, Jiménez Sáiz S, Sampaio J, Marques MC. Effects of knowing the task duration on players' pacing patterns during soccer small-sided games. J Sports Sci. 2018;36:116-122.

13. Gabbett TJ, Mulvey M. Time-motion analysis of small sided training games and competition in elite women soccer players. J Strength Cond Res. 2008;22:543-552.

14. Gaudino P, Iaia FM, Alberti G, Hawkins RD, Strudwick AJ, Gregson W. Systematic bias between running speed and metabolic power data in elite soccer players: influence of drill type. Int J Sports Med. 2014;35:489-93.

15. Gaudino P, Iaia FM, Alberti G, Strudwick AJ, Atkinson G, Gregson W. Monitoring training in elite soccer players: systematic bias between running speed and metabolic power data. Int J Sports Med. 2013;34:963-968.

16. Goto H, Morris JG, Nevill ME. Motion analysis of U11 to U16 elite English premier league academy players. J Sports Sci. 2015;33:1-11.

17. Halouani J, Chtourou H, Gabbett T, Chaouachi A, Chamari K. Small-sided games in team sports training: A brief review. J Strength Cond Res. 2014;28:3594-3618.

18. Harley JA, Barnes CA, Portas M et al. Motion analysis of match-play in elite U12 to U16 age-group soccer players. J Sports Sci. 2010;28:1391-1397.

19. Highton J, Mullen T, Norris J, Oxendale C, Twist, C. Energy expenditure derived from micro-technology is not suitable for assessing internal load in collision-based activities. Int J Sports Physiol Perform. 2016;20:957-961. 
20. Hill-Haas SV, Coutts AJ, Dawson BT, Rowsell GJ. Time-motion characteristics and physiological responses of small-sided games in elite youth players: The influence of player number and rule changes. J Strength Cond Res. 2010;24:2149-2156.

21. Hill-Haas S, Coutts A, Rowsell G, Dawson B. Generic versus small sided game training in soccer. Int J Sports Med. 2009;30:636-642.

22. Hill-Haas SV, Dawson BT, Coutts AJ, Rowsell GJ. Physiological responses and timemotion characteristics of various small-sided soccer games in youth players. J Sports Sci. 2009;27:1-8.

23. Hill-Haas SV, Dawson B, Impellizzeri FM, Coutts AJ. Physiology of small-sided games training in football a systematic review. Sports Med. 2011;41:199-220.

24. Hill-Haas SV, Rowsell GJ, Dawson BT, Coutts AJ. Acute physiological responses and time-motion characteristics of two small-sided training regimes in youth soccer players. $J$ Strength Cond Res. 2009;23:111-115.

25. Impellizzeri FM, Marcora SM, Castagna C et al. Physiological and performance effects of generic versus specific aerobic training in soccer players. Int J Sports Med. 2006;27:483-492.

26. Johnston, RJ, Watsford, ML, Kelly, SJ, Pine, MJ, Spurrs RW. Validity and interunit reliability of $10 \mathrm{~Hz}$ and $15 \mathrm{~Hz}$ GPS units for assessing athlete movement demands. $J$ Strength Cond Res. 2014;28:1649-1655.

27. Jones S, Drust B. Physiological and Technical Demands of $4 \mathrm{v} 4$ and $8 \mathrm{v} 8$ games in elite youth soccer players. Kinesiology. 2007;39:150-156.

28. Lacome M, Simpson BM, Cholley Y, Lambert P, Buchheit M. Small-Sided Games in Elite Soccer: Does One Size Fits All? Int J Sports Physiol Perform. 2018;13(5):568-576.

29. Mero A, Komi PV. Electromyographic activity in sprinting at speeds ranging from submaximal to supra-maximal. Med Sci Sports Exerc. 1987;19:266-274. 
30. Mohr M, Krustrup P, Bangsbo J. Match performance of high-standard soccer players with special reference to development of fatigue. J Sports Sci. 2003;21:519-528.

31. Ngo JK, Tsui MC, Smith AW, Carling C, Chan GS, Wong del P. The effects of manmarking on work intensity in small-sided soccer games. J Sports Sci Med. 2012;1:109114.

32. Olthof SBH, Frencken WGP, Lemmink KAPM. Match-derived relative pitch area changes the physical and team tactical performance of elite soccer players in small-sided soccer games. J Sports Sci. 2017;10:1-7.

33. Osgnach C, Poser S, Bernardini R, Rinaldo R, di Prampero PE. Energy cost and metabolic power in elite soccer: A new match analysis approach. Med Sci Sports Exerc. 2010;42:170-178.

34. Rampinini E, Impellizzeri FM, Castagna $C$ et al. Factors influencing physiological responses to small-sided soccer games. J Sports Sci. 2007;25:659-666.

35. Rebelo AN, Silva P, Rago V, Barreira D, Krustrup P. Differences in strength and speed demands between 4v4 and 8v8 small-sided football games. J Sports Sci. 2016;34:22462254.

36. Reilly T. An ergonomics model of the soccer training process. J Sports Sci. 2005;23:561572.

37. Reilly T, Williams AM, Nevill A, Franks A. A multidisciplinary approach to talent identification in soccer. J Sports Sci. 2000;18:695-702.

38. Sassi A, Stefanescu A, Menaspa' P, Bosio A, Riggio M, Rampinini E. The cost of running on natural grass and artificial turf surfaces. J Strength Cond Res. 2011;25:606-611.

39. Stevens TG, De Ruiter CJ, Beek PJ, Savelsbergh GJ. Validity and reliability of 6-a-side small-sided game locomotor performance in assessing physical fitness in football players. J Sports Sci. 2016;34:527-534. 
424 40. The Fédération Internationale de Football Association (FIFA). Laws of the game. http://www.fifa.com/development/education-and-technical/referees/laws-of-thegame.html. Accessed March 15, 2018.

41. Varley MC, Fairweather IH, Aughey RJ. Validity and reliability of GPS for measuring instantaneous velocity during acceleration, deceleration, and constant motion. J Sports Sci. 2012;30:121-127.

42. Waldron M, Worsfold P, Twist C, Lamb K. Concurrent validity and test-retest reliability of a global positioning system (GPS) and timing gates to assess sprint performance variables. J Sports Sci. 2011;29:1613-1619.

433

434

435 
436

437

438

439

440

441

442

443

444

445

446

447

448

449

450

451

452

453

454

455

456

457

458

\section{FIGURE LEGENDS}

Figure 1. Total distance covered during SSGs and 11M. Significantly different at ${ }^{*} \mathrm{p}<0.05$ **P $<0.01 .{ }^{* * *} \mathrm{P}<0.001$. SSGS $=$ small small-sided game; SSGM $=$ medium small-sided game; SSGL = Large small-sided game; $11 \mathrm{M}=11$-a-side match.

Figure 2. TS during SSGs and 11M. Significantly different at ${ }^{*} \mathrm{p}<0.05 * * \mathrm{P}<0.01 . * * * \mathrm{P}<$ 0.001. SSGS = small small-sided game; SSGM = medium small-sided game; SSGL = Large small-sided game; $11 \mathrm{M}=11$-a-side match, $\mathrm{TS}=$ distance covered at a speed $\geq 4.3 \mathrm{~m} \cdot \mathrm{s}^{-1}$.

Figure 3. TP during SSGs and 11M. Significantly different at ${ }^{*} \mathrm{p}<0.05 * * \mathrm{P}<0.01 .{ }^{* * *} \mathrm{P}<$ 0.001. SSGS = small small-sided game; SSGM = medium small-sided game; SSGL = Large small-sided game; $11 \mathrm{M}=11$-a-side match, $\mathrm{TP}=$ distance covered at metabolic power of $\geq 20$ $\mathrm{W} \cdot \mathrm{kg}^{-1}$.

Figure 4. Magnitude of difference between TS and TP in SSGs and 11M. Significantly different at ${ }^{*} \mathrm{p}<0.05 * * \mathrm{P}<0.01 . * * * \mathrm{P}<0.001 . \mathrm{SSGS}=$ small small-sided game; SSGM $=$ medium small-sided game; SSGL = Large small-sided game; $11 \mathrm{M}=11$-a-side match, $\mathrm{TS}=$ distance covered at a speed $\geq 4.3 \mathrm{~m} \cdot \mathrm{s}^{-1}, \mathrm{TP}=$ distance covered at metabolic power of $\geq 20$ $\mathrm{W} \cdot \mathrm{kg}^{-1}$. 
Table 1. Established characteristics of SSGs and 11M.

\begin{tabular}{lcccc}
\hline & SSGS & SSGM & SSGL & $11 \mathrm{M}$ \\
\hline Pitch length (m) & 39 & 55 & 78 & 105 \\
Pitch width (m) & 25 & 36 & 50 & 68 \\
Playing area $\left(\mathrm{m}^{2}\right)$ & 975 & 1980 & 3900 & 7140 \\
Area per player $\left(\mathrm{m}^{2}\right)$ & 81 & 165 & 325 & 325 \\
Duration (min) & 35 & 35 & 35 & 35 \\
Goalkeepers & Yes & Yes & Yes & Yes \\
Offside rule & No & No & No & Yes \\
\hline
\end{tabular}

460 
Figure 1

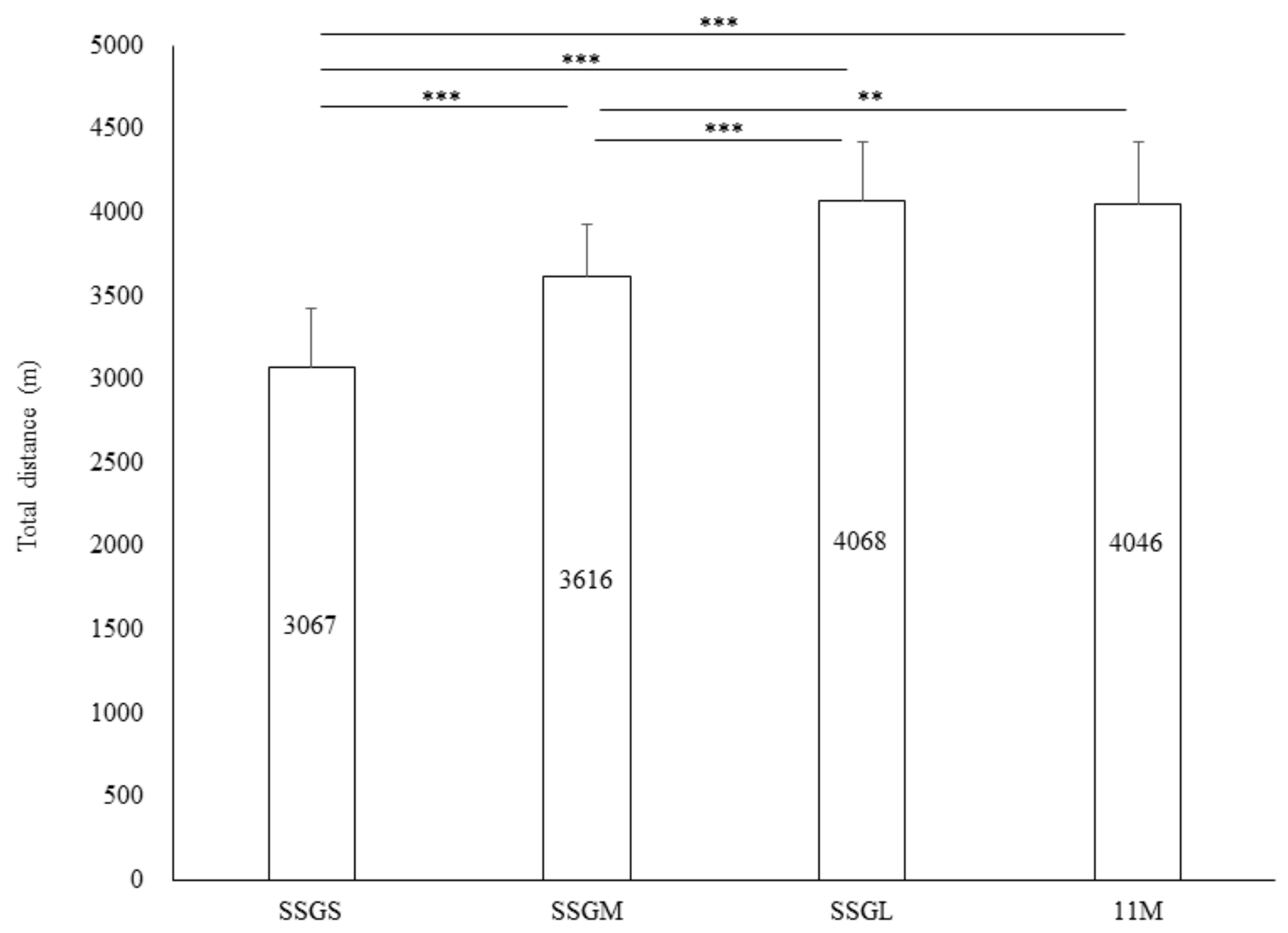


Figure 2

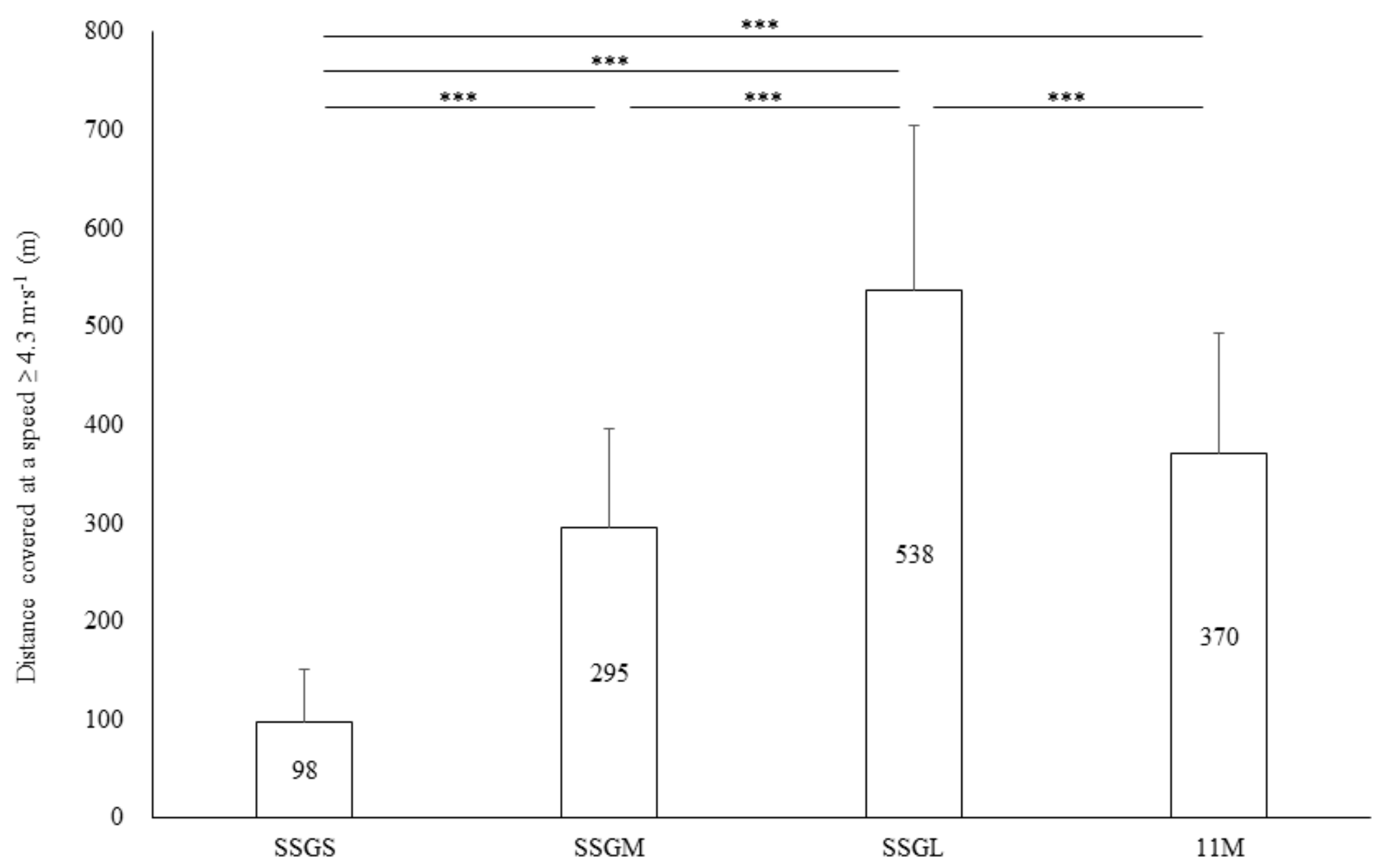


Figure 3

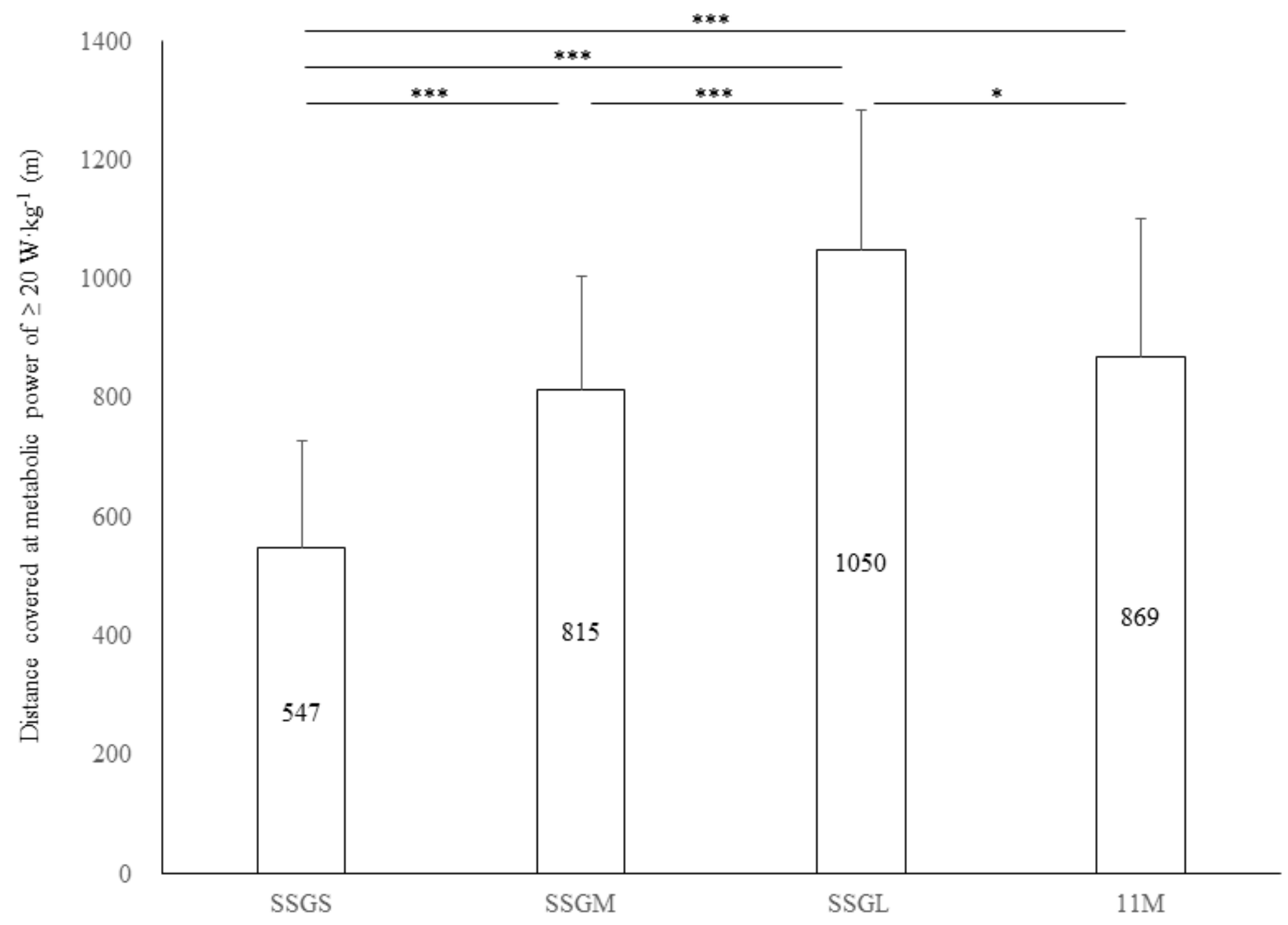


Figure 4

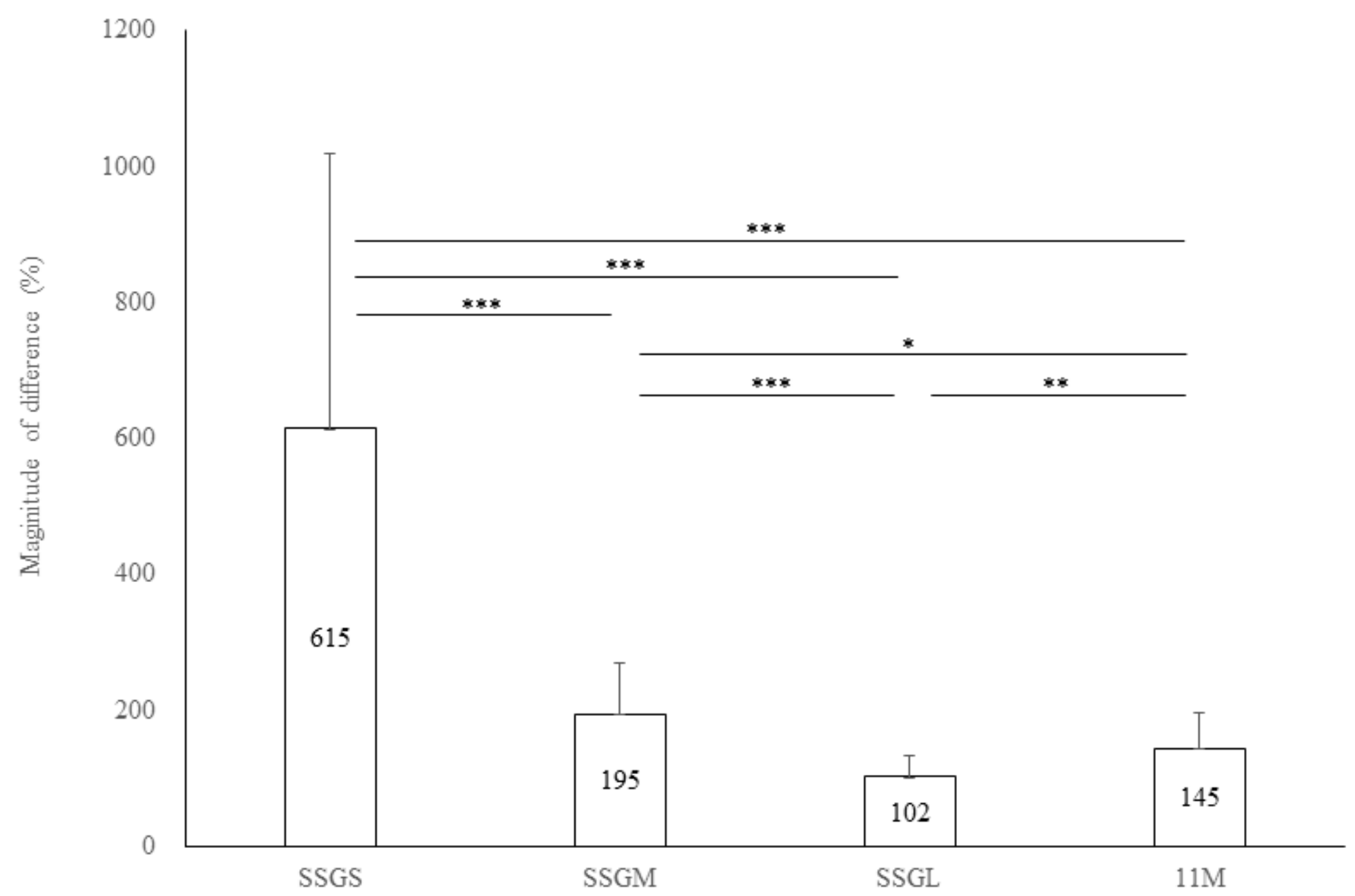

